\title{
Intrinsic ratchets: A Hamiltonian approach
}

\author{
A.V. Plyukhin* \\ Department of Mathematics, Saint Anselm College, Manchester, New Hampshire 03102, USA
}

(Dated: March 18, 2021)

\begin{abstract}
An asymmetric Brownian particle subjected to an external time-dependent force may acquire a net drift velocity, and thus operate as a motor or ratchet, even if the external force is represented by an unbiased time-periodic function or by a zero-centered noise. For an adequate description of such ratchets, a conventional Langevin equation linear in the particle's velocity is insufficient, and one needs to take into account the first nonlinear correction to the dissipation force which emerges beyond the weak coupling limit. We derived microscopically the relevant nonlinear Langevin equation by extending the standard projection operation technique beyond the weak coupling limit. The particle is modeled as a rigid cluster of atoms and its asymmetry may be geometrical, compositional (when a cluster is composed of atoms of different types), or due to a combination of both factors. The drift velocity is quadratic in the external force's amplitude and increases with decreasing the force's frequency (for a periodic force) and inverse correlation time (for a fluctuating force). The maximum value of the drift velocity is independent on the particle's mass and achieved in the adiabatic limit, i.e. for an infinitely slow change of the external field.
\end{abstract}

\section{INTRODUCTION}

Intrinsic ratchets, first studied and christened in Ref. [1], are Brownian motors based on an asymmetric Brownian particle subjected to a time-dependent unbiased external force $F_{e x}(t)$. The latter may be either deterministic (e.g., sinusoidally-varying), or noisy with zero mean. An orientation of the particle with respect to the direction of $F_{e x}$ is assumed to be fixed, which implies that the particle moves in one dimension, perhaps along a track or channel. The latter serves merely as a geometric constraint and, unlike models of flashing and rocking ratchets [2, 3], does not impose a tilted periodic potential in order to break the spatial symmetry of the system. For intrinsic ratchets the spatial symmetry is broken by the (intrinsic) asymmetry of the particle itself. On the other hand, the external time-dependent force $F_{e x}(t)$ prevents the particle from reaching thermal equilibrium with the surrounding thermal bath and thus breaks the microscopic dynamical symmetry of detailed balance. For granular ratchets (not considered here) which interact with molecules via dissipative collisions, and therefore by construction are in a nonequilibrium state, the external force is not required [4, 5].

Considering minimalistic requirements (a single isothermal bath, no need for an external spatially periodic and/or biased potential) and the omnipresence of fluctuating electric forces (particularly thermal electric noise in living cells $[6,[7])$ which may serve as an external drive $F_{e x}(t)$, it is tempting to think of intrinsic ratchets as one of the closest approximations to a perpetual motion machine of the second kind that Nature allows.

Besides satisfying conditions of non-equilibrium and spatial asymmetry, it is generally believed that any machine rectifying thermal fluctuations must operate in a nonlinear regime [2, 3]. Again, in contrast to many other models, the nonlinearity of intrinsic ratchets originates not from an external potential but rather from a nonlinear correction to a dissipation force exerted by the thermal bath on a Brownian particle. In this sense intrinsic ratchets are "intrinsic" not only because of geometric asymmetry of the particle but also because of the inherent dissipative nonlinearity of Brownian motion. Clearly, a conventional linear Langevin equation for the particle momentum $P$

$$
\frac{d}{d t} P(t)=-\gamma_{0} P(t)+F_{e x}(t)+\xi(t)
$$

cannot produce a long-lived average drift $\langle P(t)\rangle$ if the external force $F_{e x}(t)$ is unbiased and the thermal noise force $\xi(t)$ exerted by the bath is zero-centered. On the other hand, the microscopic theory of Brownian motion teaches us that the linear Langevin equation (11) is actually an approximation which may be insufficient in certain cases. Namely, equation (11) can be derived, under certain assumptions, from the underlying microscopic dynamics in the weak coupling limit, i.e. in leading order in a small mass ratio parameter $\lambda=\sqrt{m / M} \ll 1$, where $m$ is the mass of a molecule of the bath and $M$ is that of the particle, see e.g. 8|. To higher orders in $\lambda$, additional forces nonlinear in the particle momentum emerge in the Langevin equation [8-12]. Though typically small, these nonlinear dissipation

*Electronic address: aplyukhin@anselm.edu 
forces may lead to new physical effects which do not show up in the weak coupling limit [1, 11,13$]$. Such effects, the drift of intrinsic ratchets being one of them, originate technically from the coupling of the first moment $\langle P(t)\rangle$ of the particle's momentum to the second $\left\langle P^{2}(t)\right\rangle$ and/or higher moments. On the other hand, the linear Langevin equation (11) implies a closed equation for $\langle P(t)\rangle$ and is clearly insufficient.

The coupling of the first and higher moments of a targeted variable is the most distinctive feature of stochastic dynamics beyond the weak coupling limit. It can be addressed with an approach based on either the Langevin 11, [12, 18] or master equation [14, 22], the latter is closely related to the $1 / \Omega$ expansion method of van Kampen [15 17]. While being approximate, the description of classical Langevin dynamics beyond the weak coupling limit has proved in many studies to be accurate and consistent 1, 10 14, 19 22]. In particular, it enjoys thermalization toward a correct equilibrium distribution at any order of perturbation theory [16 18]. An alternative approach to the same class of problems is developed in 23, 24].

We shall show that for an asymmetric Brownian particle in a homogeneous thermal bath, by going one order of $\lambda$ higher than the weak coupling limit, one obtains, instead of (1), a nonlinear Langevin equation

$$
\frac{d}{d t} P(t)=-\gamma_{0} P(t)-\gamma_{1}\left(P^{2}(t)-\left\langle P^{2}\right\rangle_{e}\right)+F_{e x}(t)+\xi(t),
$$

where $\left\langle P^{2}\right\rangle_{e}$ is the equilibrium second moment of the particle momentum, and $\xi(t)$ is a zero-centered noise. The corresponding equation for the first moment reads

$$
\frac{d}{d t}\langle P(t)\rangle=-\gamma_{0}\langle P(t)\rangle-\gamma_{1}\left[\left\langle P^{2}(t)\right\rangle-\left\langle P^{2}\right\rangle_{e}\right]+F_{e x}(t) .
$$

The second moment appears here multiplied by the coefficient $\gamma_{1}$ which is of higher order in $\lambda$. It is therefore sufficient to complement Eq. (3) with the equation for the second moment in leading order in $\lambda$

$$
\frac{d}{d t}\left\langle P^{2}(t)\right\rangle=-2 \gamma_{0}\left[\left\langle P^{2}(t)\right\rangle-\left\langle P^{2}\right\rangle_{e}\right]+2 F_{e x}(t)\langle P(t)\rangle,
$$

which can be derived from the linear Langevin equation (11), see section IV for details. It was shown in [1] that the system of coupled equations (3) and (4) predicts a systematic drift of the particle even if the external force $F_{e x}(t)$ is time-periodic and unbiased. For example, for a harmonic drive $F_{e x}(t)=F_{0} \sin (\omega t)$ a calculation based on Eqs. (3) and (4) gives the average momentum $\langle P(t)\rangle$ which oscillates with the frequency $\omega$ in such a manner that its positive and negative semi-periods do not completely compensate each other, see the inset in Fig. 3 below. As a result, the net time-averaged particle's momentum and velocity does not vanish. A systematic drift also may take place if the external force $F_{e x}(t)$ is not a regular time-periodic function but a zero-centered nonequilibrium noise. A key parameter which determines a direction and magnitude of the drift is the nonlinear dissipation coefficient $\gamma_{1}$ in (2). For a symmetric Brownian particle in a uniform thermal bath $\gamma_{1}$ vanishes and so does the drift.

In Ref. [1], the nonlinear term $-\gamma_{1}\left[\left\langle P^{2}(t)\right\rangle-\left\langle P^{2}\right\rangle_{e}\right]$ was introduced in Eq. (3) phenomenologically based on an intuitively appealing requirement that at low perturbation order the coupling of the first two moments $\langle P(t)\rangle$ and $\left\langle P^{2}(t)\right\rangle$ must be linear and vanish in equilibrium. In Refs. [19, 20, 22], equations (3) and (44) were derived kinetically within a mesoscopic model where the ratchet is modeled as a structureless asymmetric Brownian object interacting with molecules of the bath via elastic collisions. Explicit expressions for the coefficient $\gamma_{1}$ were derived for two [19, 20] and three-dimensional [22] convex-shaped Brownian objects. While providing an important insight, these model calculations are based on specific assumptions that the thermal bath is an ideal gas of non-interacting molecules, and that collisions of molecules with a ratchet are instantaneous, binary, and uncorrelated.

The main goal of the present paper is to derive the nonlinear Langevin equation (2) for an asymmetric Brownian particle microscopically from the underlying Hamiltonian dynamics. We shall do this with a standard projection operator technique extended in two ways. First, we model a Brownian particle not as a point-like object (which of course cannot be asymmetric), but rather as a cluster of atoms connected by rigid bonds and interacting with molecules of the surrounding bath via spherically symmetric and short-ranged potentials. Second, we shall go one perturbation order higher than the standard weak coupling approximation, which is of order $\lambda^{2}$, retaining terms up to order $\lambda^{3}$. The outcome of such derivation will be the Langevin equation (2) with the dissipation coefficients $\gamma_{0}$ and $\gamma_{1}$ expressed in terms of microscopic correlation functions. The expression for the nonlinear dissipation coefficient $\gamma_{1}$ qualifies as a new fluctuation-dissipation relation, additional to the conventional one for the linear dissipation coefficient $\gamma_{0}$.

One advantage of such a microscopic approach is that it offers a more natural framework to describe ratchets based on nanoscale molecular systems (proteins, nucleic acids, lipids, molecular assemblies, etc.) whose asymmetry is often not merely geometric but due to the inherent structural inhomogeneity. Although in this paper we use the approximation of rigid bonds and do not consider the internal dynamics of ratchets, it appears that the theory can be 


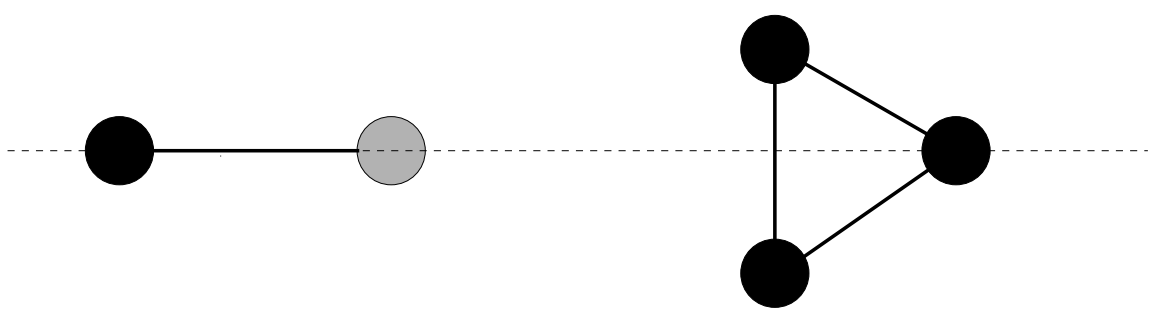

FIG. 1: Simplest atomic clusters with different types of asymmetry. Left: a dimer made of two different atoms (structural asymmetry). Right: a trimer made of three identical atoms (geometrical asymmetry).

readily extended in that direction too. Another benefit of a microscopic approach is that it shows that the nonlinear Langevin equation (21) is quite generic and does not imply specific assumptions and restrictions (e.g., of a thermal bath made of an ideal gas) typically imposed in model calculations. Expressing the nonlinear dissipation coefficient $\gamma_{1}$ in terms of a microscopic correlation function (rather than specific parameters of a particular system) allows one to analyze the problem within a more general and unifying framework based on fluctuation-dissipation relations. The price for this generality is that relevant correlation functions are hard to evaluate analytically and to be determined from experiment or simulation.

Besides a microscopic derivation of nonlinear Langevin equation (2) addressed in sections II and III, we shall also exploit this equation to evaluate the net drift of intrinsic ratchets activated by harmonic and fluctuating external forces in section IV. Results of a few illustrative molecular dynamics simulations of the ratchets based on simple atomic clusters (a dimer and trimer) are presented in section V. Summarizing remarks are collected in section VI.

\section{MODEL}

We model an asymmetric Brownian particle as a cluster of rigidly connected material points, referred below as "atoms", enumerated by index $\nu$, and having masses $M_{\nu}$. We shall use the terms "particle" and "cluster" interchangeably. The total mass of the cluster $M=\sum_{\nu} M_{\nu}$ is much larger than the mass $m$ of a molecule of the surrounding thermal bath. The particle's asymmetry may be geometrical (related to the cluster's shape), structural (when the cluster is composed of different atoms), see Fig. 1, or due to a combination of both factors. While each atom is assumed to interact with bath molecules through a spherically symmetric potential, equipotential surfaces of the total potential created by all atoms of a cluster may, of course, lack any symmetry.

The position vectors of particle's atoms $\mathbf{R}_{\nu}(t)$ are convenient to express as

$$
\mathbf{R}_{\nu}(t)=\mathbf{R}(t)+\mathbf{a}_{\nu}
$$

where $\mathbf{R}(t)=\sum_{\nu} M_{\nu} \mathbf{R}_{\nu}(t) / M$ is the position vector of the particle's center of mass, and $\mathbf{a}_{\nu}$ are position vectors of the atoms in the center-of-mass reference frame. The particle is constrained to move along the $x$-axis keeping fixed its shape and orientation, the former due to the rigidity of bonds, the latter due to being attached to an ideal track, or several parallel tracks, inducing no friction. Accordingly, the vectors $\left\{\mathbf{a}_{\nu}\right\}$, as well as components $Y$ and $Z$ of the center-of-mass position vector $\mathbf{R}=(X, Y, Z)$ are fixed and do not change with time. Then the particle's motion is characterized by a single conjugate coordinate-momentum pair, namely the $x$-component of the center-of-mass position vector $\mathbf{R}(t)$ and the total momentum of the cluster,

$$
X=\sum_{\nu} \frac{M_{\nu} X_{\nu}}{M}, \quad P=\sum_{\nu} M_{\nu} \dot{X}_{\nu}
$$

The problem is therefore formally equivalent to that of a point-like Brownian particle moving in one dimension and interacting with the bath via an asymmetric effective potential.

The overall Hamiltonian of the particle and the thermal bath has the form

$$
H=\frac{P^{2}}{2 M}+H_{0}+U_{e x}(t)
$$

The external potential $U_{e x}(t)$ is assumed to act on each atom of the particle, but not on molecules of the bath,

$$
U_{e x}(t)=\sum_{\nu} u_{e x}\left(\mathbf{R}_{\nu}, t\right)=\sum_{\nu} u_{e x}\left(\mathbf{R}+\mathbf{a}_{\nu}, t\right)
$$


The term $H_{0}=H_{0}(X)$ is the Hamiltonian of bath molecules in the the potential of the Brownian particle when the center of mass of the former held fixed at the position with $x$-coordinate equal to $X$,

$$
H_{0}(X)=\sum_{i} \frac{\mathbf{p}_{i}^{2}}{2 m}+V+\Phi(X)
$$

where $V=\sum_{i>i^{\prime}} v\left(\mathbf{r}_{i}-\mathbf{r}_{i^{\prime}}\right)$ is the potential for interaction of bath molecules, and

$$
\Phi(X)=\sum_{\nu, i} \phi_{\nu}\left(\mathbf{R}_{\nu}-\mathbf{r}_{i}\right)=\sum_{\nu, i} \phi_{\nu}\left(\mathbf{R}+\mathbf{a}_{\nu}-\mathbf{r}_{i}\right)
$$

is the potential for interaction of bath molecules and atoms of the particle when the center-of-mass of the latter has position $\mathbf{R}=(X, Y, Z)$. In the above expressions, $\left\{\mathbf{r}_{i}, \mathbf{p}_{i}\right\}$ denote position vectors and momenta of bath molecules, the subscript $i$ refers to bath molecules and $\nu$ to atoms of the particle. The notation $\phi_{\nu}$ implies that different atoms of the cluster may interact with the bath via different potentials. The potentials $v$ and $\phi_{\nu}$ are assumed to be spherically symmetric and short-ranged.

The Liouville operator $L=\{\cdots, H\}$ of the closed system "the particle plus bath" is a Poisson bracket with the overall Hamiltonian $H$. It splits naturally in two parts

$$
L(t)=L_{0}+L_{1}^{\prime}(t)
$$

The first part $L_{0}=\left\{\cdots, H_{0}\right\}$ describes dynamics of the bath in the potential field of a fixed Brownian particle,

$$
L_{0}=\sum_{i}\left(\frac{\mathbf{p}_{i}}{m} \cdot \frac{\partial}{\partial \mathbf{r}_{i}}+\mathbf{f}_{i} \cdot \frac{\partial}{\partial \mathbf{p}_{i}}\right)
$$

where $\mathbf{f}_{i}=-\partial(V+\Phi) / \partial \mathbf{r}_{i}$ is a force on $i$ th bath molecule. The second part involves derivatives with respect to the particle's variables,

$$
L_{1}^{\prime}(t)=\frac{P}{M} \frac{\partial}{\partial X}+\left[F+F_{e x}(t)\right] \frac{\partial}{\partial P},
$$

where $F=-\partial \Phi / \partial X$ and $F_{e x}=-\partial U_{e x} / \partial X$ are the $x$-projection of the forces exerted on the particle by the bath and external field, respectively. We temporarily denote $L_{1}^{\prime}$ with a prime because another form for this term will be introduced shortly.

The standard assumptions and settings of the microscopic theory of Brownian motion are assumed to be hold. Initial conditions for dynamical variables of the bath are random and distributed according to the equilibrium canonical distribution with the Hamiltonian $H_{0}$, inverse temperature $\beta=1 /\left(k_{B} T\right)$, and partition function $Z_{0}$,

$$
\rho_{0}=Z_{0}^{-1} \exp \left(-\beta H_{0}\right), \quad Z_{0}=\int \exp \left(-\beta H_{0}\right) \prod_{i} d \mathbf{r}_{i} d \mathbf{p}_{i} .
$$

When averaged over distribution (14), the force $\mathbf{F}_{\nu}=-\partial \phi_{\nu} / \partial \mathbf{R}_{\nu}$ from the bath on atom $\nu$ vanishes, $\left\langle\mathbf{F}_{\nu}\right\rangle=$ $\int \rho_{0} \mathbf{F}_{\nu} \prod_{i} d \mathbf{r}_{i} d \mathbf{p}_{i}=0$, and so does the total force $\mathbf{F}=\sum_{\nu} \mathbf{F}_{\nu}$ on the cluster, $\langle\mathbf{F}\rangle=\sum_{\nu}\left\langle\mathbf{F}_{\nu}\right\rangle=0$. In order to describe the constrained motion of a rigid cluster we need only the $x$-projection of the total force $\mathbf{F}$, denoted above by $F$ and referred from now on simply as "force", which of course is also zero on average, $\langle F\rangle=0$, whether the cluster is symmetric or not.

Next, the particle's momentum is expected to be close to the equilibrium value $\sqrt{M / \beta}$, which is $\sqrt{M / m}$ time larger than the equilibrium value of the bath molecule's momentum $p_{e}=\sqrt{m / \beta}$. Then it is convenient to work with a scaled momentum of the particle

$$
P_{*}=\lambda P, \quad \lambda=\sqrt{m / M} \ll 1
$$

which on average is of the same order of magnitude as $p_{e}$. When written in terms of $P_{*}$, the Liouville operator takes the form

$$
L(t)=L_{0}+\lambda L_{1}(t)
$$

where $L_{0}$ is still given by (12) while $L_{1}=\lambda^{-1} L_{1}^{\prime}$ reads

$$
L_{1}(t)=\frac{P_{*}}{m} \frac{\partial}{\partial X}+\left[F+F_{e x}(t)\right] \frac{\partial}{\partial P_{*}} .
$$


The form (16) is more convenient than (11) for developing a proper perturbation technique since the dependence on the small parameter $\lambda$ in (16) is explicit.

Starting with the equation of motion for the particle's scaled momentum

$$
\dot{P}_{*}(t)=\lambda F(t)+\lambda F_{e x}(t)
$$

our goal is to transform this equation into a Langevin form by partitioning the term $F(t)$, representing the force exerted on the particle by the bath, into a dissipative and fluctuating parts. While many steps of the derivation are standard, others are less so. In order to make the paper self-contained, we shall present the derivation in full.

As a preparation, one notes that the equation of motion for the force $\dot{F}(t)=L(t) F(t)$ with the initial condition $F(0)=F$ is equivalent to the integral equation $F(t)=F+\int_{0}^{t} d \tau L(\tau) F(\tau)$, which can be solved by iteration:

$$
F(t)=\left\{1+\int_{0}^{t} d \tau L(\tau)+\int_{0}^{t} d \tau_{1} \int_{0}^{\tau_{1}} d \tau_{2} L\left(\tau_{1}\right) L\left(\tau_{2}\right)+\cdots\right\} F
$$

This may be expressed concisely as

$$
F(t)=\overrightarrow{\exp }\left(\int_{0}^{t} L(\tau) d \tau\right) F
$$

in terms of the time-ordered exponential propagator

$$
\overrightarrow{\exp }\left(\int_{0}^{t} L(\tau) d \tau\right) \equiv T_{+}\left\{\sum_{n=0}^{\infty} \frac{1}{n !}\left(\int_{0}^{t} L(\tau) d \tau\right)^{n}\right\}=1+\int_{0}^{t} d \tau L(\tau)+\int_{0}^{t} d \tau_{1} \int_{0}^{\tau_{1}} d \tau_{2} L\left(\tau_{1}\right) L\left(\tau_{2}\right)+\cdots
$$

where the time-ordering operator $T_{+}$rearranges the product of time-dependent operators in such a way that time arguments decrease from left to right. For example, $T_{+}\left\{L\left(\tau_{1}\right) L\left(\tau_{2}\right)\right\}$ equals $L\left(\tau_{1}\right) L\left(\tau_{2}\right)$ if $\tau_{1}>\tau_{2}$ and $L\left(\tau_{2}\right) L\left(\tau_{1}\right)$ otherwise. As a result, operators earlier in time act before operators at later times. We shall also need an operator

$$
\overleftarrow{\exp }\left(-\int_{0}^{t} L(\tau) d \tau\right) \equiv T_{-}\left\{\sum_{n=0}^{\infty} \frac{1}{n !}\left(-\int_{0}^{t} L(\tau) d \tau\right)^{n}\right\}=1-\int_{0}^{t} d \tau L(\tau)+\int_{0}^{t} d \tau_{1} \int_{0}^{\tau_{1}} d \tau_{2} L\left(\tau_{2}\right) L\left(\tau_{1}\right)-\cdots
$$

where the time-ordering operator $T_{-}$makes later times operators to appear not on the left (as $T_{+}$does), but on the right. The second equalities in (21) and (22) can be proved by interchanging integration variables, see e.g. Ref. [25]. Note that in our notations an arrow over exponentials indicates a direction of decreasing time arguments, which appears not to be a generally accepted convention in the literature.

Operators (21) and (22) commute and are inverse to each other,

$$
\overrightarrow{\exp }\left(\int_{0}^{t} L(\tau) d \tau\right) \overleftarrow{\exp }\left(-\int_{0}^{t} L(\tau) d \tau\right)=\overleftarrow{\exp }\left(-\int_{0}^{t} L(\tau) d \tau\right) \overleftrightarrow{\exp }\left(\int_{0}^{t} L(\tau) d \tau\right)=1
$$

We shall also need the differentiation properties

$$
\frac{d}{d t} \overrightarrow{\exp }\left(\int_{0}^{t} L(\tau) d \tau\right)=L(t) \overrightarrow{\exp }\left(\int_{0}^{t} L(\tau) d \tau\right), \quad \frac{d}{d t} \overleftarrow{\exp }\left(-\int_{0}^{t} L(\tau) d \tau\right)=-\overleftarrow{\exp }\left(-\int_{0}^{t} L(\tau) d \tau\right) L(t),
$$

which follow directly from the second equalities in (21) and (22).

In the next section we shall use expression (20) as a starting point to project out the bath variables from the force $F(t)$ with a projection operator and perturbation techniques. The necessity of time-ordered exponentials is dictated, of course, by the non-commuting of Liouville operators $L(t)$ at different times. We shall see, however, that up to order $\lambda^{3}$ (which is sufficient for our purposes) the time dependent term $F_{e x}(t) \frac{\partial}{\partial P_{*}}$ in the Liouville operator does not actually affect the structure of the dissipative force in the Langevin equation. Knowing that in advance, one could neglect the time dependence of $L(t)$ and work with, instead of (20), with the much simpler expression $F(t)=e^{L t} F$. Up to order $\lambda^{3}$, such an ad-hoc simplified approach gives a correct partitioning of the bath-induced force $F(t)$. It is hard to see, however, another way to justify this insight but working out (as we do below) the exact expression (20). 


\section{NONLINEAR LANGEVIN EQUATION}

In this section we exploit a projection operator technique in the form originally developed in Ref. [8] for a point-like Brownian particle and to order $\lambda^{2}$. Applying the approach to a rigid asymmetric cluster of point-like atoms and extending a perturbation procedure to order $\lambda^{3}$ we shall be able to derive microscopically the nonlinear Langevin equation (2), which suffices to describe the operation of intrinsic ratchets.

As mentioned above, the idea is to start with the exact expression (20) for the force $F(t)$ exerted on the particle by the bath and to partition it into a dissipative (depending only on the particle's momentum) and fluctuating parts. To this end, we need a generalization of the familiar operator identity

$$
e^{(A+B) t}=e^{A t}+\int_{0}^{t} e^{A(t-\tau)} B e^{(A+B) \tau} d \tau
$$

to the case when operators $A$ and $B$ are time-dependent and do not self-commute at different times. Such generalization has the form

$$
\begin{gathered}
\overrightarrow{\exp }\left(\int_{0}^{t}[A(s)+B(s)] d s\right)=\overrightarrow{\exp }\left(\int_{0}^{t} A(s) d s\right) \\
+\int_{0}^{t} \overrightarrow{\exp }\left(\int_{0}^{t} A(s) d s\right) \overleftarrow{\exp }\left(-\int_{0}^{\tau} A(s) d s\right) B(\tau) \overrightarrow{\exp }\left(\int_{0}^{\tau}[A(s)+B(s)] d s\right) d \tau
\end{gathered}
$$

This identity can be verified by multiplying both sides from the left by $\overleftarrow{\exp }\left(-\int_{0}^{t} A(s) d s\right)$ and then differentiating with respect to $t$, also taking into account properties (23) and (24).

Using (26) with $A=L(t)$ and $B=-\mathcal{P} L(t)$ (with yet an arbitrary operator $\mathcal{P}$ ), the force $F(t)=\overrightarrow{\exp }\left(\int_{0}^{t} L(s) d s\right) F$ can be expressed as

$$
F(t)=F_{*}(t)+\overrightarrow{\exp }\left(\int_{0}^{t} L(s) d s\right) \int_{0}^{t} \overleftarrow{\exp }\left(-\int_{0}^{\tau} L(s) d s\right) \mathcal{P} L(\tau) F_{*}(\tau) d \tau
$$

where

$$
F_{*}(t)=\overrightarrow{\exp }\left(\int_{0}^{t} \mathcal{Q} L(s) d s\right) F, \quad \mathcal{Q}=1-\mathcal{P} .
$$

With a properly chosen operator $\mathcal{P}$, expression (27) will eventually represent the desirable partition of $F(t)$, with the first term $F_{*}(t)$ playing the role of the fluctuating Langevin force, while the second term will develop into a dissipative force.

We define $\mathcal{P}$ as an operator of averaging (of an arbitrary dynamical variable $A$ ) over initial values of bath variables $\mathbf{r}=\left\{\mathbf{r}_{i}\right\}, \mathbf{p}=\left\{\mathbf{p}_{i}\right\}$ with the canonical distribution (14),

$$
\mathcal{P} A=\langle A\rangle=\int \rho_{0} A d \mathbf{r} d \mathbf{p} .
$$

Then both $\mathcal{P}$ and $\mathcal{Q}=1-\mathcal{P}$ are projection operators, $\mathcal{P} \mathcal{P}=\mathcal{P}, \mathcal{Q} \mathcal{Q}=\mathcal{Q}$, and are orthogonal

$$
\mathcal{P} \mathcal{Q}=\mathcal{Q P}=0 .
$$

The vanishing of the equilibrium average force exerted by the bath on the particle can now be expressed in the form

$$
\langle F\rangle=\mathcal{P} F=0 .
$$

As follows from (28)-(31), the fluctuating component $F_{*}(t)$ is zero-centered too,

$$
\left\langle F_{*}(t)\right\rangle=\mathcal{P} F_{*}(t)=0 .
$$

The major benefit of choosing $\mathcal{P}$ in the form (29) comes from the relation

$$
\mathcal{P} L_{0}=\int \rho_{0} L_{0}(\ldots) d \mathbf{r} d \mathbf{p}=0
$$


where the Liouville operator of the bath $L_{0}$ is given by (12). This allows one to eliminate the explicit dependence on bath variables in the second term in (27):

$$
\mathcal{P} L(t) F_{*}(t)=\mathcal{P}\left[L_{0}+\lambda L_{1}(t)\right] F_{*}(t)=\lambda \mathcal{P} L_{1}(t) F_{*}(t)=\lambda \frac{P_{*}}{m} \mathcal{P} \frac{\partial}{\partial X} F_{*}(t)+\lambda \frac{\partial}{\partial P_{*}} \mathcal{P}\left[F+F_{e x}(t)\right] F_{*}(t) .
$$

Moreover, since $\mathcal{P} F_{\text {ex }}(t) F_{*}(t)=F_{\text {ex }}(t) \mathcal{P} F_{*}(t)=0$, the dependence on the external force is eliminated as well,

$$
\mathcal{P} L(t) F_{*}(t)=\lambda \frac{P_{*}}{m} \mathcal{P} \frac{\partial}{\partial X} F_{*}(t)+\lambda \frac{\partial}{\partial P_{*}} \mathcal{P} F F_{*}(t) .
$$

Here the first term on the right hand side can be worked out with a useful relation

$$
\frac{\partial}{\partial X} \mathcal{P} F_{*}(t)=0=\beta \mathcal{P} F F_{*}(t)+\mathcal{P} \frac{\partial}{\partial X} F_{*}(t)
$$

to get

$$
\mathcal{P} L(t) F_{*}(t)=\lambda\left(\frac{\partial}{\partial P_{*}}-\frac{\beta P_{*}}{m}\right) \mathcal{P} F F_{*}(t)=\lambda\left(\frac{\partial}{\partial P_{*}}-\frac{\beta P_{*}}{m}\right)\left\langle F F_{*}(t)\right\rangle .
$$

Substitution of this into (27) gives for the force exerted by the bath the following, and still exact, expression

$$
F(t)=F_{*}(t)+\lambda \overrightarrow{\exp }\left(\int_{0}^{t} L(s) d s\right) \int_{0}^{t} \overleftarrow{\exp }\left(-\int_{0}^{\tau} L(s) d s\right)\left(\frac{\partial}{\partial P_{*}}-\frac{\beta P_{*}}{m}\right)\left\langle F F_{*}(\tau)\right\rangle d \tau
$$

The next step is to expand the fluctuating force

$$
F_{*}(t)=\overrightarrow{\exp }\left(\int_{0}^{t} \mathcal{Q} L(s) d s\right) F=\overrightarrow{\exp }\left(\int_{0}^{t}\left[L_{0}+\lambda \mathcal{Q} L_{1}(s)\right] d s\right) F
$$

in powers of $\lambda$. The first two terms will suffice our purpose,

$$
F_{*}(t)=F_{0}(t)+\lambda F_{1}(t)+O\left(\lambda^{2}\right) .
$$

Applying iteratively identity (26) with $A=L_{0}$ and $B(t)=\lambda \mathcal{Q} L_{1}(t)$ to (39), one gets

$$
F_{0}(t)=e^{L_{0} t} F, \quad F_{1}(t)=\int_{0}^{t} e^{L_{0}(t-\tau)} \mathcal{Q} L_{1}(\tau) F_{0}(\tau) d \tau .
$$

The term $F_{0}(t)$ has a meaning of the force exerted by the bath on a fixed (or infinitely heavy) particle and does not depend on the particle's momentum $P_{*}$. On the other hand, the term $F_{1}(t)$ originates from the particle's motion and depends on $P_{*}$. This dependence must be explicitly extracted in order to work out the second term in (38).

In view of expansion (40), to first order in $\lambda$ the force (38) acquires the form

$$
F(t)=F_{*}(t)-\frac{\lambda \beta}{m} \overrightarrow{\exp }\left(\int_{0}^{t} L(s) d s\right) \int_{0}^{t} \overleftarrow{\exp }\left(-\int_{0}^{\tau} L(s) d s\right) P_{*}\left\langle F F_{0}(\tau)\right\rangle d \tau .
$$

For a low density bath, one can neglect the coupling of the force on the particle and the slow hydrodynamic modes of the bath [26] and to apply the Markovian ansatz $\left\langle F F_{0}(t)\right\rangle \rightarrow \delta(t) \int_{0}^{\infty}\left\langle F F_{0}(t)\right\rangle d t$. Then expression (42) is further simplified to

$$
F(t)=F_{*}(t)-\frac{\lambda \beta}{m} P_{*}(t) \int_{0}^{\infty}\left\langle F F_{0}(t)\right\rangle d t
$$

Substitution of this into the equation of motion (18) yields the linear Langevin equation

$$
\dot{P}_{*}(t)=-\lambda^{2} \zeta_{0} P_{*}(t)+\lambda F_{*}(t)+\lambda F_{e x}(t)
$$

with the dissipating coefficient

$$
\zeta_{0}=\frac{\beta}{m} \int_{0}^{\infty}\left\langle F F_{0}(t)\right\rangle d t
$$


In terms of the unscaled momentum $P=P_{*} / \lambda$, Eq. (44) takes the standard form (1)

$$
\dot{P}(t)=-\gamma_{0} P(t)+F_{*}(t)+F_{e x}(t),
$$

with the dissipating coefficient

$$
\gamma_{0}=\lambda^{2} \zeta_{0}=\frac{\beta}{M} \int_{0}^{\infty}\left\langle F F_{0}(t)\right\rangle d t
$$

and zero-centered fluctuating force, $\left\langle F_{*}(t)\right\rangle=0$. Thus in lowest order in $\lambda$ the Langevin equation for an asymmetric rigid cluster has the same form as for a point-like Brownian particle and cannot account for the operation of intrinsic ratchets.

More interesting, and in fact sufficient for our purpose, is an approximation of the exact expression (38) for $F(t)$ to order $\lambda^{2}$. Since the second term in (38) contains the factor $\lambda$, it suffices to substitute there a linear approximation $F_{*}(t)=F_{0}(t)+\lambda F_{1}(t)$. The component $F_{1}(t)$ is given by (41), or more explicitly

$$
F_{1}(t)=\int_{0}^{t} d \tau e^{L_{0}(t-\tau)} \mathcal{Q}\left(\frac{P_{*}}{m} \frac{\partial}{\partial X}+\left[F+F_{e x}(t)\right] \frac{\partial}{\partial P_{*}}\right) F_{0}(\tau) .
$$

Since $F_{0}(t)$ does not depend on $P_{*}$, this is reduced to

$$
F_{1}(t)=\frac{P_{*}}{m} \int_{0}^{t} d \tau\left(e^{L_{0}(t-\tau)} \frac{\partial}{\partial X} F_{0}(\tau)-\mathcal{P} \frac{\partial}{\partial X} F_{0}(\tau)\right) .
$$

Here, similar to (36), $\mathcal{P} \frac{\partial}{\partial X} F_{0}(t)=-\beta\left\langle F F_{0}(t)\right\rangle$, which gives

$$
F_{1}(t)=\frac{P_{*}}{m} \int_{0}^{t} d \tau\left(e^{L_{0}(t-\tau)} \frac{\partial}{\partial X} F_{0}(\tau)+\beta\left\langle F F_{0}(\tau)\right\rangle\right) .
$$

Note that to first order in $\lambda$ the fluctuating force $F_{*}(t) \approx F_{0}(t)+\lambda F_{1}(t)$ is independent of the external force $F_{\text {ex }}(t)$ (this is not so for higher perturbation orders).

With expression (50) for $F_{1}(t)$ at hand, we can work out the correlation function $\left\langle F F_{*}(t)\right\rangle=\left\langle F F_{0}(t)\right\rangle+\lambda\left\langle F F_{1}(t)\right\rangle$ in (38) as follows:

$$
\left\langle F F_{*}(t)\right\rangle=C_{0}(t)+\lambda \frac{P_{*}}{m} C_{1}(t),
$$

where

$$
C_{0}(t)=\left\langle F F_{0}(t)\right\rangle, \quad C_{1}(t)=\int_{0}^{t} d \tau\left\langle F e^{L_{0}(t-\tau)} \frac{\partial}{\partial X} F_{0}(\tau)\right\rangle .
$$

It can be proved with a symmetry argument (see the Appendix) that for a symmetric particle in a uniform bath the correlation $C_{1}(t)$ vanishes identically (in that case the first non-zero correction to the weak-coupling approximation $\left\langle F F_{*}(t)\right\rangle=C_{0}(t)$ is of order $\left.\lambda^{2}\right)$. On the other hand, for an asymmetric particle the correlation $C_{1}(t)$ does not vanish in general and ultimately is responsible for the operation of intrinsic ratchets.

Substitution of (51) into (38) yields for $F(t)$ an approximation of order $\lambda^{2}$

$$
F(t)=F_{*}(t)-\frac{\lambda \beta}{m} \overrightarrow{\exp }\left(\int_{0}^{t} L(s) d s\right) \int_{0}^{t} \overleftarrow{\exp }\left(-\int_{0}^{\tau} L(s) d s\right)\left\{C_{0}(\tau) P_{*}+\frac{\lambda}{m} C_{1}(\tau)\left(P_{*}^{2}-\frac{m}{\beta}\right)\right\} d \tau
$$

In the Markovian approximation $C_{i}(t) \rightarrow \delta(t) \int_{0}^{\infty} C_{i}(t) d t, i=0,1$, this expression acquires the form

$$
F(t)=F_{*}(t)-\frac{\lambda \beta}{m} P_{*}(t) \int_{0}^{\infty} C_{0}(\tau) d \tau-\frac{\lambda^{2} \beta}{m^{2}}\left(P_{*}^{2}(t)-\frac{m}{\beta}\right) \int_{0}^{\infty} C_{1}(\tau) d \tau .
$$

Finally, substitution of this expression into the equation of motion (18) yields the nonlinear Langevin equation of order $\lambda^{3}$

$$
\dot{P}_{*}(t)=-\lambda^{2} \zeta_{0} P_{*}(t)-\lambda^{3} \zeta_{1}\left(P_{*}^{2}(t)-\left\langle P_{*}^{2}\right\rangle_{e}\right)+\lambda F_{*}(t)+\lambda F_{e x}(t),
$$


where $\left\langle P_{*}^{2}\right\rangle_{e}=m / \beta$ is the equilibrium value of the scaled momentum squared, the dissipative constants are

$$
\zeta_{0}=\frac{\beta}{m} \int_{0}^{\infty} C_{0}(t) d t, \quad \zeta_{1}=\frac{\beta}{m^{2}} \int_{0}^{\infty} C_{1}(t) d t,
$$

the correlation functions $C_{0}(t)$ and $C_{1}(t)$ are given by (52), and $F_{*}(t)$ is a zero-centered noise, $\left\langle F_{*}(t)\right\rangle=0$. Adopting the Markovian approximation we assume that the characteristic time $\tau_{0}$ of correlations $C_{0}(t)$ and $C_{1}(t)$ is distinctly shorter than both the period of the external force $F_{e x}(t)$ and the momentum relaxation time $\tau_{p}=1 / \lambda^{2} \zeta_{0}$. Then Eq. (55) corresponds to a coarse-grain description with a time resolution $\Delta t \gg \tau_{0}$.

In terms of the unscaled momentum $P=P_{*} / \lambda$, the Langevin equation (55) has the form (2),

$$
\dot{P}(t)=-\gamma_{0} P(t)-\gamma_{1}\left(P^{2}(t)-\left\langle P^{2}\right\rangle_{e}\right)+F_{*}(t)+F_{e x}(t),
$$

where $\left\langle P^{2}\right\rangle_{e}=M / \beta$ is the equilibrium value of the square momentum, and dependence on $\lambda$ is now absorbed in the rescaled dissipation coefficients

$$
\gamma_{0}=\lambda^{2} \zeta_{0}=\frac{\beta}{M} \int_{0}^{\infty} C_{0}(t) d t, \quad \gamma_{1}=\lambda^{4} \zeta_{1}=\frac{\beta}{M^{2}} \int_{0}^{\infty} C_{1}(t) d t .
$$

Relations (56) or (58) are ought to be viewed as fluctuation-dissipation relations for an asymmetric Brownian particle. Note again that for a symmetric particle the correlation function $C_{1}(t)$ and the nonlinear dissipation coefficient $\gamma_{1}$ vanish and the first nonlinear correction is of order $\lambda^{4}$ and cubic in the momentum [9, 12].

In the next section we shall prefer to evaluate the particle's drift using the Langevin equation for the scaled momentum in the form (55), which involves the small parameter $\lambda$ explicitly.

\section{EVALUATION OF DRIFT}

Let us derive a set of two coupled equations for the first two moments of the particle's scaled momentum

$$
A(t)=\left\langle P_{*}(t)\right\rangle, \quad B(t)=\left\langle P_{*}^{2}(t)\right\rangle-\left\langle P_{*}^{2}\right\rangle_{e} .
$$

As in the previous section, the angular brackets denote averaging over initial values of bath variables with the distribution (14). Taking average of the Langevin equation (55) and assuming that the external force $F_{e x}(t)$ is uniform (does not depend on the particle's position), one obtains the first equation

$$
\dot{A}(t)=-\lambda^{2} \zeta_{0} A(t)-\lambda^{3} \zeta_{1} B(t)+\lambda F_{e x}(t) .
$$

A systematic directional motion of the particle is expected to emerge as a result of the coupling of $A(t)$ and $B(t)$. Since the latter appears in (60) multiplied by $\lambda^{3}$, it is sufficient to complement (60) with a familiar equation for the second moment to order $\lambda^{2}$,

$$
\dot{B}(t)=-2 \lambda^{2} \zeta_{0} B(t)+2 \lambda F_{e x}(t) A(t) .
$$

The easiest way to obtain this equation is multiplying the linear Langevin equation (44) by $2 P_{*}(t)$, taking average, and taking into account that $\left\langle P_{*}(t) F_{0}(t)\right\rangle=\lambda \zeta_{0} m / \beta=\lambda \zeta_{0}\left\langle P_{*}^{2}\right\rangle_{e}$ The latter relation can be directly verified using an explicit solution $P_{*}(t)$ of the linear equation (44), the Markovian anzatz for the autocorrelation of $F_{0}(t)$, and the fluctuation-dissipation relation (45).

Eqs. (60) and (61) form a closed system of linear equations for the first two moments of $P_{*}$. They are equivalent to Eqs. (3) and (4) of the Introduction and to those exploited in Ref. 1]. Since we are looking for a stationary solution, the specific choice of initial conditions is immaterial. Assuming for simplicity $A(0)=B(0)=0$, the solution of (60) can be written as

$$
A(t)=\lambda a_{1}(t)+\lambda^{3} a_{2}(t)
$$

where

$$
a_{1}(t)=\int_{0}^{t} d t^{\prime} e^{-\lambda^{2} \zeta_{0}\left(t-t^{\prime}\right)} F_{e x}\left(t^{\prime}\right), \quad a_{2}(t)=-\zeta_{1} \int_{0}^{t} d t^{\prime} e^{-\lambda^{2} \zeta_{0}\left(t-t^{\prime}\right)} B\left(t^{\prime}\right)
$$


and the solution of (61) is

$$
B(t)=2 \lambda \int_{0}^{t} d t^{\prime} e^{-2 \lambda^{2} \zeta_{0}\left(t-t^{\prime}\right)} F_{e x}\left(t^{\prime}\right) A\left(t^{\prime}\right)
$$

Equations (62) and (64) can be uncoupled by substituting in (64) an approximation $A(t)=\lambda a_{1}(t)+\lambda^{3} a_{2}(t) \approx \lambda a_{1}(t)$ which gives

$$
B(t)=2 \lambda^{2} \int_{0}^{t} d t^{\prime} e^{-2 \lambda^{2} \zeta_{0}\left(t-t^{\prime}\right)} F_{e x}\left(t^{\prime}\right) \int_{0}^{t^{\prime}} d t^{\prime \prime} e^{-\lambda^{2} \zeta_{0}\left(t^{\prime}-t^{\prime \prime}\right)} F_{e x}\left(t^{\prime \prime}\right)
$$

With this expression for $B(t)$, the second equation of (63) yields for $a_{2}(t)$

$$
a_{2}(t)=-2 \lambda^{2} \zeta_{1} \int_{0}^{t} d t_{1} e^{-\lambda^{2} \zeta_{0}\left(t-t_{1}\right)} \int_{0}^{t_{1}} d t_{2} e^{-2 \lambda^{2} \zeta_{0}\left(t_{1}-t_{2}\right)} F_{e x}\left(t_{2}\right) \int_{0}^{t_{2}} d t_{3} e^{-\lambda \zeta_{0}\left(t_{2}-t_{3}\right)} F_{e x}\left(t_{3}\right) .
$$

As a result, for the average scaled momentum $\left\langle P_{*}\right\rangle=A=\lambda a_{1}+\lambda^{3} a_{2}$ one gets

$$
\begin{aligned}
\left\langle P_{*}(t)\right\rangle & =\lambda \int_{0}^{t} d t_{1} e^{-\lambda^{2} \zeta_{0}\left(t-t_{1}\right)} F_{e x}\left(t_{1}\right) \\
& -2 \lambda^{5} \zeta_{1} \int_{0}^{t} d t_{1} e^{-\lambda^{2} \zeta_{0}\left(t-t_{1}\right)} \int_{0}^{t_{1}} d t_{2} e^{-2 \lambda^{2} \zeta_{0}\left(t_{1}-t_{2}\right)} F_{e x}\left(t_{2}\right) \int_{0}^{t_{2}} d t_{3} e^{-\lambda^{2} \zeta_{0}\left(t_{2}-t_{3}\right)} F_{e x}\left(t_{3}\right) .
\end{aligned}
$$

This result is obtained under the assumption that the second term in (62) or (67) is much smaller than the first one. Contrary to what expressions (62) or (67) may suggest, the smallness of $\lambda$ alone does not guarantee the validity of this approximation since both terms may actually be of the same order in $\lambda$; this will be shown below explicitly for specific forms of $F_{e x}(t)$. On the other hand, it is clear from (67) that the approximation does hold for sufficiently small values of $\zeta_{1}$ and/or amplitude of the external force. The precise consistency condition will be formulated shortly.

Let us re-write the above result for the unscaled momentum $P=\lambda^{-1} P_{*}$ as a sum of two contributions,

$$
\begin{gathered}
\langle P(t)\rangle=\langle P(t)\rangle_{0}+\langle P(t)\rangle_{1}, \\
\langle P(t)\rangle_{0}=\int_{0}^{t} d t_{1} e^{-\gamma_{0}\left(t-t_{1}\right)} F_{e x}\left(t_{1}\right), \\
\langle P(t)\rangle_{1}=-2 \gamma_{1} \int_{0}^{t} d t_{1} e^{-\gamma_{0}\left(t-t_{1}\right)} \int_{0}^{t_{1}} d t_{2} e^{-2 \gamma_{0}\left(t_{1}-t_{2}\right)} F_{e x}\left(t_{2}\right) \int_{0}^{t_{2}} d t_{3} e^{-\gamma_{0}\left(t_{2}-t_{3}\right)} F_{e x}\left(t_{3}\right),
\end{gathered}
$$

where we use the rescaled dissipation coefficients $\gamma_{0}=\lambda^{2} \zeta_{0}$ and $\gamma_{1}=\lambda^{4} \zeta_{1}$ as they appear in the Langevin equation (57) for $P$ and given explicitly by (58). Here the first term $\langle P(t)\rangle_{0}$, representing the linear response to the external force, can be obtained from the linear Langevin equation (46). It contributes to the particle's net drift only if $F_{e x}(t)$ is biased, i.e. when the time-average of $F_{e x}(t)$ is non-zero. The second term $\langle P(t)\rangle_{1}$ represents a nonlinear response contribution originating from the coupling of $\langle P\rangle$ and $\left\langle P^{2}\right\rangle$ to higher order in $\lambda$. Being quadratic in the external force $F_{e x}(t)$, this term can produce a directional drift of the particle even when $F_{e x}(t)$ is unbiased. The drift may be characterized by the net momentum $P_{n e t}$, or net velocity $V_{\text {net }}=P_{n e t} / M$, defined as a time-average, which we shall denoted by an overbar, of the ensemble average $\langle P(t)\rangle$,

$$
P_{n e t}=\overline{\langle P(t)\rangle}=\lim _{T \rightarrow \infty} \frac{1}{T} \int_{0}^{T}\langle P(t)\rangle d t
$$

Consider first a harmonic external force $F_{e x}(t)=F_{0} \sin \omega t$. In this case, from (68)-(71) one finds for $t \gg 1 / \gamma_{0}$

$$
P_{n e t}=-\frac{\gamma_{1}}{2 \gamma_{0}^{3}} \frac{F_{0}^{2}}{1+\left(\omega / \gamma_{0}\right)^{2}}
$$

This expression originates entirely from the nonlinear response term $\langle P(t)\rangle_{1}$ given by $(\overline{700}), P_{n e t}=\overline{\langle P(t)\rangle}_{1}$. The linear response term has a form

$$
\langle P(t)\rangle_{0}=\frac{F_{0}}{\gamma_{0}^{2}+\omega^{2}}\left\{\gamma_{0} \sin \omega t+\omega\left(e^{-\gamma_{0} t}-\cos \omega t\right)\right\}
$$


and vanishes after averaging over time in the long-time limit, ${\overline{\langle P(t)\rangle_{0}}}=0$.

Recall that we obtained the results (68) and (72) under the assumption that the nonlinear contribution to the average momentum is much smaller than the linear one, $\langle P(t)\rangle_{1} \ll\langle P(t)\rangle_{0}$. According to (58), the dissipation coefficients scale with the particle's mass as

$$
\gamma_{0} \sim M^{-1}, \quad \gamma_{1} \sim M^{-2}
$$

Then it follows from (72)-(74) that for the low-frequency domain $\omega \ll \gamma_{0}$, both linear $\langle P(t)\rangle_{0}$ and nonlinear $\langle P(t)\rangle_{1} \sim$ $P_{n e t}$ contributions scale in the same way (linearly) with $M$. As was already noted, this means that the condition of small mass ratio, $\lambda \ll 1$, is not sufficient for the consistency of the above approach. A comparison of Eqs. (72) and (73) shows that one has to require an additional condition

$$
\frac{\gamma_{1}}{\gamma_{0}^{2}} F_{0} \ll 1, \quad \text { for } \quad \omega \ll \gamma_{0}
$$

According to (74), this constraint does not involve the particle's mass $M$, and implies a small value of the nonlinear dissipation coefficient $\gamma_{1}$ and/or of the external force amplitude $F_{0}$. On the other hand, for the high-frequency domain instead of (75) one gets

$$
\frac{\gamma_{1}}{\gamma_{0} \omega} F_{0} \ll 1, \quad \text { for } \quad \omega \gg \gamma_{0}
$$

Since $\gamma_{1} / \gamma_{0} \sim M^{-1}$, this condition can be satisfied for sufficiently small $\lambda$.

The quantity $\gamma_{0}$ has a meaning of the inverse time for the particle's momentum relaxation, $\gamma_{0}=1 / \tau_{p}$, and for a micro-meter sized Brownian object in water has a value of order about $10^{7} s^{-1}$. Then according to (72) the frequencyindependent maximum value of the net momentum

$$
P_{\max }=-\frac{\gamma_{1}}{2 \gamma_{0}^{3}} F_{0}^{2}
$$

corresponds to the frequency of long-radio waves. Remarkably, according to (74) and (77), the maximum net velocity $V_{\max }=P_{\max } / M$ does not depend on the particle's mass.

Consider also the case when the external force is a stationary random processes $F_{e x}(t)=F_{e x}(t \mid \theta)$ whose specific realizations depends on a random parameter (or a set of parameters) $\theta$. One may think of $F_{e x}(t \mid \theta)$, for instance, as of a force generated by randomly moving external charges with a set $\theta$ of initial coordinates and momenta. Whatever physical system is responsible for generating the force $F_{e x}(t \mid \theta)$, we shall assume that it is not in equilibrium with the thermal bath, and that the average of $F_{e x}(t \mid \theta)$ over $\theta$, which we denote as $\left\langle F_{e x}(t)\right\rangle_{\theta}$, is zero.

For this case, the momentum averaged over the bath $\langle P(t)\rangle$ is still given by expressions (68)-(70), but now we define the net momentum $P_{n e t}$ as an average of $\langle P(t)\rangle$ over $\theta$, rather than over time. Again, since $\left\langle F_{\text {ex }}(t)\right\rangle_{\theta}=0$ the linear response $\langle P(t)\rangle_{0}$ does not contribute to the net momentum, while taking the average over $\theta$ of the expression (70) for the nonlinear response $\langle P(t)\rangle_{1}$ gives

$$
P_{n e t}=-2 \gamma_{1} \int_{0}^{t} d t_{1} e^{-\gamma_{0}\left(t-t_{1}\right)} \int_{0}^{t_{1}} d t_{2} e^{-2 \gamma_{0}\left(t_{1}-t_{2}\right)} \int_{0}^{t_{2}} d t_{3} e^{-\gamma_{0}\left(t_{2}-t_{3}\right)}\left\langle F_{\text {ex }}\left(t_{2}\right) F_{e x}\left(t_{3}\right)\right\rangle_{\theta} .
$$

Suppose the auto-correlation function of the fluctuating external force $F_{e x}(t)$ is exponential,

$$
\left\langle F_{e x}(t) F_{e x}\left(t^{\prime}\right)\right\rangle_{\theta}=F_{0}^{2} \exp \left(-\frac{\left|t-t^{\prime}\right|}{\tau_{c}}\right) .
$$

Then for $t \gg 1 / \gamma_{0}$ the net momentum (78) acquires the form

$$
P_{n e t}=-\frac{\gamma_{1}}{\gamma_{0}^{3}} \frac{F_{0}^{2}}{1+1 /\left(\gamma_{0} \tau_{c}\right)}
$$

In this expression, the noise correlation time $\tau_{c}$ plays a role similar to the inverse frequency of a harmonic force, and the validity conditions are similar to (75) and (76). As a function of $\tau_{c}$, the net momentum reaches the maximal value in the limit of $\gamma_{0} \tau_{c} \gg$ 1, i.e. when the correlation time of $F_{e x}(t)$ is much longer than the momentum relaxation time $\tau_{p}=1 / \gamma_{0}$. 


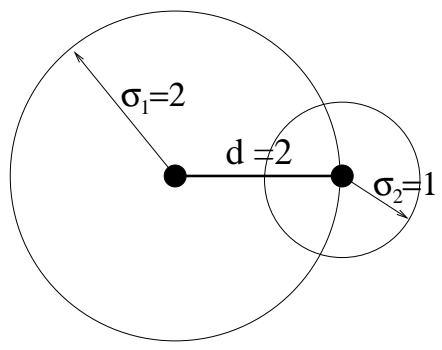

A

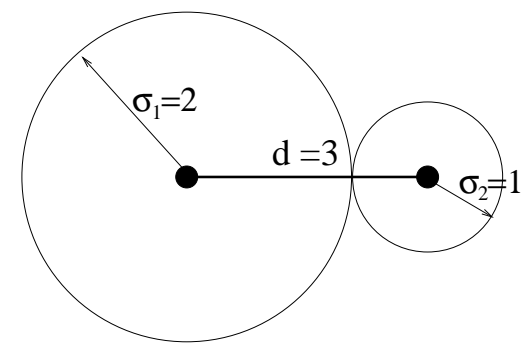

B

FIG. 2: Two dimer configurations with the same radii of the atom-molecule interaction spheres, $\sigma_{1}=2$ and $\sigma_{2}=1$, and different lengths $d$. Configuration $A$ with $d=2$ (on the left) is characterized by a significant overlapping of the interaction spheres and is found to have a larger value of the net drift velocity.

\section{SIMULATION}

In this section we describe molecular dynamics simulations of intrinsic ratchets based on two- and three-atom clusters immersed in the two-dimensional ideal gas. Our goal here is to provide simple illustrations, rather than quantitative verification of theoretical predictions of the previous sections. Such a verification would require an explicit theoretical evaluation of dissipation coefficients $\gamma_{0}$ and $\gamma_{1}$ with relations (58), which is beyond the scope of the present study. Instead, the simulation may be used to get some empirical insight about how $\gamma_{0}$ and $\gamma_{1}$ depend on a cluster's structure.

While the theoretical model discussed in the previous sections assumes that the ratchet's atoms are connected by rigid bonds, in our simulations we consider clusters of atoms connected by stiff harmonic bonds. We found that transport properties of ratchets only weakly depend on the value of the bond strength constant $k$, at least when the latter is sufficiently large. Therefore simulation results for stiff clusters ( $k$ is large but finite) and theoretical predictions for their rigid prototypes $(k \rightarrow \infty)$ are expected to be close.

\section{A. Dimer activated by harmonic force}

Consider a diatomic cluster (dimer) consisting of two atoms of the same mass $M_{a}=M / 2$, oriented and constrained to move along the $x$-axis. Each atom is subjected to the external harmonic drive $F_{e x}(t)=F_{0} \sin \omega t$ applied in the $x$-direction. The two atoms interact with each other via a harmonic potential

$$
U=\frac{k}{2}\left(X_{2}-X_{1}-d\right)^{2}
$$

where $d$ is the length of the unstretched dimer, $X_{1}, X_{2}$ are positions of left and right atoms, respectively. The parameters $k$ and $d$ are assumed to be sufficiently large to guarantee that $X_{2}>X_{1}$ at any time.

Atom $\nu(\nu=1,2)$ interacts with molecule $i$ of the surrounding two-dimensional bath through truncated repulsive potential of the form

$$
\phi_{\nu}\left(\mathbf{R}_{\nu}, \mathbf{r}_{i}\right)=\frac{u_{\nu}}{\alpha_{\nu}}\left(\frac{\left|\mathbf{R}_{\nu}-\mathbf{r}_{i}\right|}{\sigma_{\nu}}\right)^{-\alpha_{\nu}} h\left(\sigma_{\nu}-\left|\mathbf{R}_{\nu}-\mathbf{r}_{i}\right|\right),
$$

where $\mathbf{R}_{\nu}=\left(X_{\nu}, 0\right)$ and $\mathbf{r}_{i}=\left(x_{i}, y_{i}\right)$ are position vectors of atom $\nu$ and molecule $i$, respectively, $\sigma_{\nu}$ are the interaction radius for atom $\nu$, and $h(x)$ is the Heaviside step function. The latter makes the force corresponding to potential (82) to be zero when the distance $\left|\mathbf{R}_{\nu}-\mathbf{r}_{i}\right|$ between a molecule and atom exceeds $\sigma_{\nu}$. An artificial singularity of the force at $\left|\mathbf{R}_{\nu}-\mathbf{r}_{i}\right|=\sigma_{\nu}$ is of no consequence for our purposes.

In this setting, the dimer's asymmetry may be due to unequal potential parameters for the two atoms. As a specific example, we consider dimer configurations with the potential radius of the left atom to be two times larger than that of the right one, while other parameters of the potentials for both atoms are the same,

$$
\sigma_{1}=2 \sigma_{2}, \quad u_{1}=u_{2}=u, \quad \alpha_{1}=\alpha_{2}=\alpha .
$$

Two such configurations, denoted as $A$ and $B$, which differ by the dimer's length $d$ are shown in Fig. 2. The simulation shows that configuration $A$ with $d=\sigma_{1}=2 \sigma_{2}$ develops a larger net velocity than configuration $B$ with 


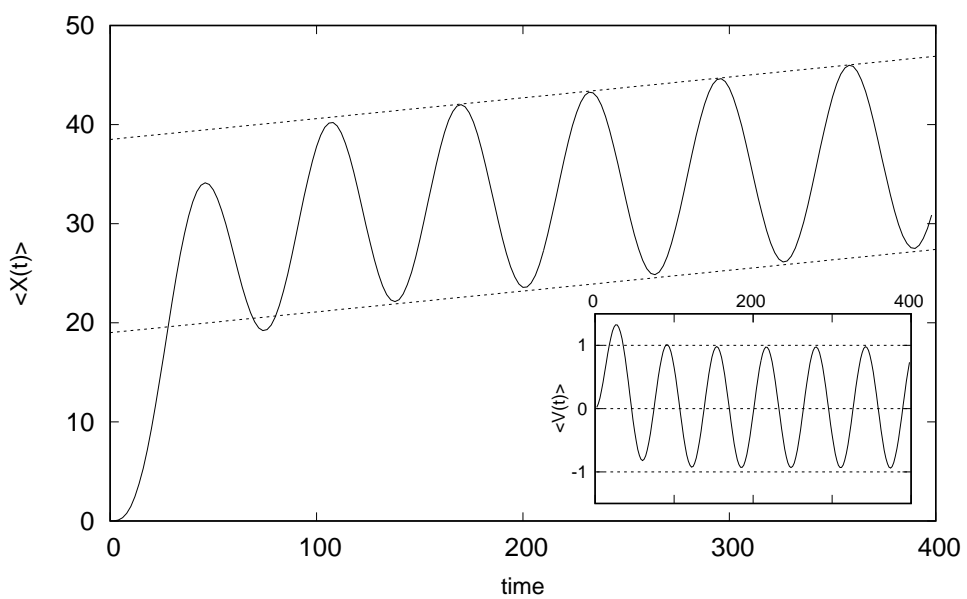

FIG. 3: Main plot: The average displacement $\langle X(t)\rangle$ of a dimer of configuration $A$ (left on Fig. 2) activated by the external harmonic force $F_{e x}(t)=F_{0} \sin \omega t$ (acting on each atom) with $F_{0}=2$ and $\omega=0.1$. For units and values of other parameters see Eq. (85) and the text above it. The slope of tangent lines (dashed lines) to minima and maxima gives the net velocity of the dimer $V_{\text {net }} \approx 0.02$. Inset: The corresponding average velocity $\langle V(t)\rangle$. The amplitude of positive peaks of the curve $\langle V(t)\rangle$ is slightly larger than that of negative ones, which results in the net motion to the right. The net velocity $V_{n e t}$ can be also determined as a time average of $\langle V(t)\rangle$.

$d=1.5 \sigma_{1}=3 \sigma_{2}$. In fact, it turns out that for a low-density thermal bath configuration $A$ shows a maximum net velocity among all other configurations of type (83) with different lengths $d$. The main plot in Fig. 3 shows simulation results for the average center-of-mass displacement $\langle X(t)\rangle$ of a dimer of configuration $A$ subjected to a harmonic drive $F_{e x}(t)=F_{0} \sin \omega t$. The slope of a tangent lines to maxima or minima of the curve $\langle X(t)\rangle$ equals to the net velocity of the dimer. Alternatively, the drift may be visualized with a plot of the average center-of-mass velocity $\langle V(t)\rangle$ shown in the inset of Fig. 3. One may notice that maxima of the curve $\langle V(t)\rangle$ have slightly larger amplitudes than minima, which results in a positive net velocity. According to theoretical result (72), which we rewrite here for the net velocity in the form

$$
V_{n e t}=\frac{V_{\max }}{1+\left(\omega / \gamma_{0}\right)^{2}}, \quad V_{\max }=-\frac{\gamma_{1}}{2 M \gamma_{0}^{3}} F_{0}^{2},
$$

a positive net velocity corresponds to a negative value of the nonlinear dissipation coefficient, $\gamma_{1}<0$.

The simulation was performed for the following set of parameters: molecule-atom mass ratio $m / M_{a}=0.05$ (which corresponds to $\left.\lambda^{2}=m / M=m /\left(2 M_{a}\right)=0.025\right)$, concentration of bath molecules $\rho=0.2$, interaction exponent $\alpha=6$, energy interaction coefficient $u=1$, squared bond frequency $\Omega^{2}=k / M_{a}=1$, external force frequency $\omega=0.1$ and amplitude $F_{0}=2$. Here and below we adopt the following units of length $x_{0}$, velocity $v_{0}$, time $t_{0}$, energy $u_{0}$, and force $f_{0}$ :

$$
x_{0}=\sigma_{2}, \quad v_{0}=v_{e}, \quad t_{0}=\sigma_{2} / v_{e}, \quad u_{0}=m v_{e}^{2}, \quad f_{0}=m v_{0} / t_{0}
$$

where $v_{e}=1 / \sqrt{m \beta}$ denotes the average thermal speed of a molecule of the bath in equilibrium.

The data presented in Fig. 3 give for configuration $A$ with $d=2$ the net velocity value approximately $V_{\text {net }}=0.02$. This is about one order of magnitude smaller than the equilibrium thermal speed of the dimer $V_{e}=1 / \sqrt{M \beta}$, which in given units equals to $\lambda=\sqrt{m / M} \approx 0.16$. For configuration $B$ with $d=3$ the simulation under the same conditions shows the drift about two times slower, with $V_{n e t} \approx 0.01$. For configurations with $d>4$ and the same set of other parameters the net drift is getting very small and difficult to detect. On the other hand, decreasing $d$ from the apparently optimal value $d=2$ also results in fast decreasing of the net velocity. While theoretical result (84) for $V_{\text {net }}$ involves two parameters $\gamma_{0}$ and $\gamma_{1}$, we found that $\gamma_{0}$ depends on $d$ only weakly. Then the above-mentioned dependence of $V_{\text {net }}$ on $d$ should be attribute mostly to that of $\gamma_{1}$ and the underlying correlation $C_{1}(t)$.

With $V_{\text {net }}$ found from the simulation and the linear dissipation coefficient $\gamma_{0}$ evaluated independently (by simulating relaxation of the average momentum $\langle P(t)\rangle=P(0) e^{-\gamma_{0} t}$ in the absence of an external force), one can use Eq. (84) to evaluate the maximum net velocity $V_{\max }$ and the nonlinear dissipation coefficient $\gamma_{1}$. Let us estimate the order of the magnitude of the former: We found $\gamma_{0} \approx 0.03$ for configuration $A$ and a slightly larger (by about $10 \%$ ) value 


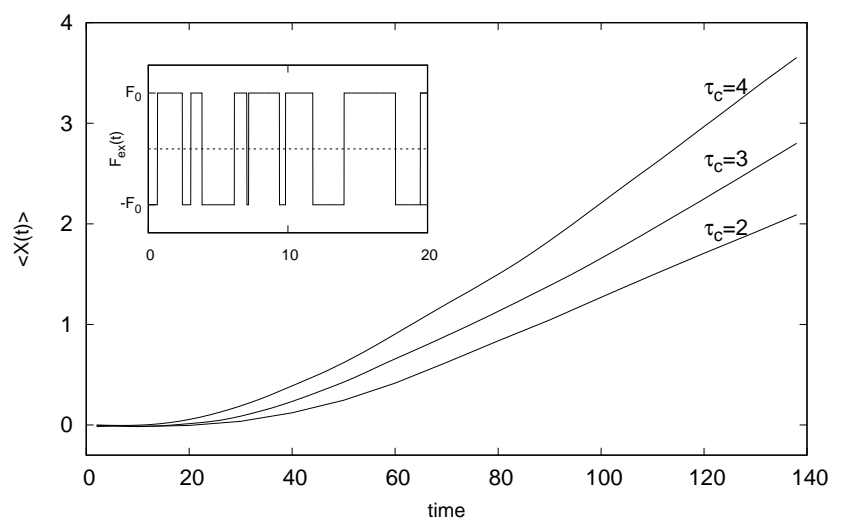

FIG. 4: Main plot: The average displacement $\langle X(t)\rangle$ of a dimer of configuration $A$ (left on Fig. 2) activated by the external telegraph noise $F_{e x}(t)$ for three values of the correlation time $\tau_{c}$. Inset: A specific realization of $F_{\text {ex }}(t)$ with $\tau_{c}=1$. The average $\langle X(t)\rangle$ is calculated over about $10^{5}$ simulation runs.

for configuration $B$. For the external force frequency $\omega=0.1$ adopted in our simulation, we have $\left(\omega / \gamma_{0}\right)^{2} \sim 10$ for both configurations. Then, according to (84), $V_{\text {net }} \sim 0.1 V_{\max }$. On the other hand, the simulation gives $V_{\text {net }} \sim 0.1 V_{e}$, and we conclude that $V_{\max }$ is comparable to the equilibrium thermal speed $V_{e}$ of the dimer. A similar estimation $V_{\max } \sim V_{e}$ was found in Ref. [1] for mesoscopic ratchets of cone shapes and in Ref. [4, 5] for granular ratchets.

A word of warning is in order here. The value $F_{0}=2$ for the external force's amplitude, adopted in our simulation, was chosen sufficiently large to make the drift easily noticeable on the plot of $\langle X(t)\rangle$ in Fig. 3. This value is likely to be too high and beyond the validity range of the theory. The latter assumes that the particle's velocity is close to its equilibrium value, $V \sim \lambda v_{e}$. In our simulation units (85), this assumption reads $V \sim \lambda \ll 1$ and, as the inset in Fig. 3 shows, for $F_{0}=2$ it is clearly violated. Therefore, if one wishes to use similar simulations for an accurate estimation of $\gamma_{1}$ and $F_{\max }$, a much weaker external field should probably be employed.

\section{B. Dimer activated by external telegraph noise}

Let the external force $F_{e x}(t)$ be a stochastic dichotomous Markov process (also known as a telegraph noise) flipping between two states $\pm F_{0}$ with a constant transition rate $k$, see the inset in Fig. 4 . The life-time $\tau$ of each of two states, i.e. the waiting time between two successive flips, is a random variable with the exponential distribution $f(\tau)=k \exp (-k \tau)$. In this case, $F_{e x}(t)$ has an exponential autocorrelation function of the form (79) with the correlation time $\tau_{c}=1 /(2 k)$, and a theoretical result for the net momentum is given by (80).

Fig. 4 shows simulation results for the displacement of the dimer of configuration A (see Fig. 2), double-averaged over initial values of bath variables and over realizations of $F_{e x}(t)$, for several values of the correlation time $\tau_{c}$. Other parameters are the same as for the simulation described in the previous subsection. With $\gamma_{0} \approx 0.03$ and $\tau_{c} \sim 1$, the parameter $\gamma_{0} \tau_{c}$ is small, $\gamma_{0} \tau_{c} \ll 1$. For this regime, Eq. (80) predicts that the net velocity can be approximated as $V_{\text {net }} \approx-\gamma_{1} \tau_{c} F_{0}^{2} /\left(M \gamma_{0}^{2}\right)$, increasing linearly with $\tau_{c}$. The data presented in Fig. 4 are qualitatively consistent with this dependence, and can be used to estimate $\gamma_{1}$. Compared to a harmonic external drive, such estimation may be more laborious since a much larger number of simulation runs is required in order to average out fluctuations (still visible on Fig. 4) of the curves $\langle X(t)\rangle$ and $\langle V(t)\rangle$.

\section{Trimer activated by harmonic force}

Consider a cluster of three atoms (trimer) in the shape (when undisturbed) of isosceles triangle with the base $2 a$, altitude $h$, and oriented with the altitude parallel to the $x$-axis, see Fig. 5. Atoms of the cluster are constrained to move (without friction) along the $x$-axis only, each along its own rail. Atoms are connected by harmonic springs with the same force constant $k$, and the internal potential energy of the cluster is

$$
U=\frac{k}{2}\left(l_{12}-l_{12}^{0}\right)^{2}+\frac{k}{2}\left(l_{13}-l_{13}^{0}\right)^{2}+\frac{k}{2}\left(l_{23}-l_{23}^{0}\right)^{2},
$$




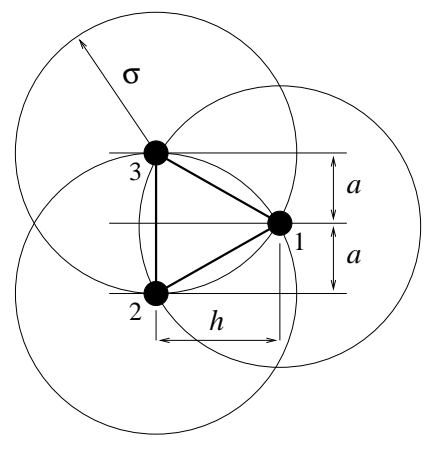

A

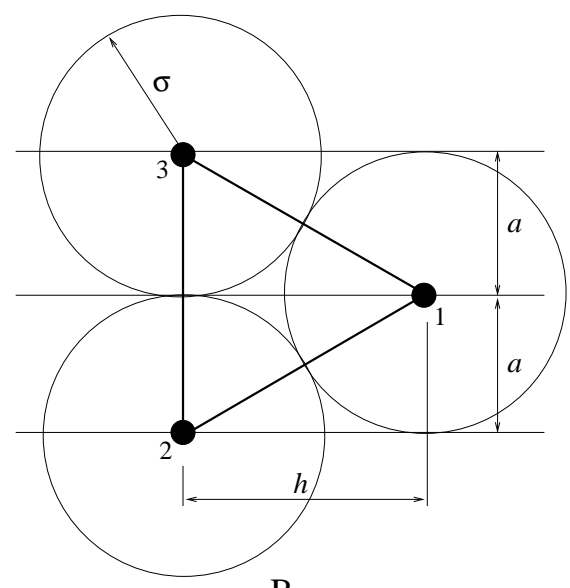

B

FIG. 5: Two equilateral trimer configurations (in equilibrium) with the same radius $\sigma$ of bath-atom interaction spheres and different side lengths $2 a$. The simulation shows that the net drift velocity of configuration $A$ with $2 a=\sigma$ (on the left) is more than three times higher than that of configuration $B$ with $a=\sigma$ (on the right), see Fig. 6 .

where $l_{i j}$ are the distances between atoms $i$ and $j$ as functions of their instantaneous $x$-coordinates,

$$
l_{12}=\sqrt{\left(X_{1}-X_{2}\right)^{2}+a^{2}}, \quad l_{13}=\sqrt{\left(X_{1}-X_{3}\right)^{2}+a^{2}}, \quad l_{23}=\sqrt{\left(X_{2}-X_{3}\right)^{2}+4 a^{2}},
$$

and $l_{i j}^{0}$ and the corresponding distances in mechanical equilibrium

$$
l_{12}^{0}=l_{13}^{0}=\sqrt{a^{2}+h^{2}}, \quad l_{23}^{0}=2 a .
$$

A value of the string constant $k$ is chosen large enough to preserve the trimer's orientation, i.e. to guarantee that at all time the vertex atom 1 is at the right of the base atoms 2 and $3, X_{1}>X_{2}, X_{3}$.

Each of three atoms $\nu=1,2,3$ interacts with a bath molecule $i$ by repulsive potential (82) of the same strength $u$, exponent $\alpha$, and radius $\sigma$,

$$
\phi_{\nu}\left(\mathbf{R}_{\nu}, \mathbf{r}_{i}\right)=\frac{u}{\alpha}\left(\frac{\left|\mathbf{R}_{\nu}-\mathbf{r}_{i}\right|}{\sigma}\right)^{-\alpha} h\left(\sigma-\left|\mathbf{R}_{\nu}-\mathbf{r}_{i}\right|\right),
$$

where the position vectors of atoms are $\mathbf{R}_{1}=\left(X_{1}, 0\right), \mathbf{R}_{2}=\left(X_{2}, a\right), \mathbf{R}_{3}=\left(X_{3},-a\right)$, and $\mathbf{r}_{i}=\left(x_{i}, y_{i}\right)$ are position vectors of bath molecules. Atoms have the same mass $M_{a}$, and the total mass $M=3 M_{a}$ of the cluster is much larger than that of a bath molecule $m$. Each atom is subjected by the same external harmonic force $F_{\text {ex }}(t)=F_{0} \sin \omega t$ applied in the $x$ direction. The simulation units are still given by (85), with $\sigma$ as a unit of length.

The simulation shows that the trimer's net velocity depends on the atomic geometry of the cluster, that is on the ratio of the altitude $h$ and the base $2 a$. The maximum drift was found for the equilateral trimer $(h / a=\sqrt{3})$. On the other hand, for a given trimer's atomic geometry the drift strongly depends on the ratio of a characteristic geometric length, say $a$, and the radius $\sigma$ of the atom-molecule interaction sphere. For an equilateral trimer, the simulation suggests that the maximal mobility is achieved for configuration $A$ with $\sigma=2 a$, see Fig. 5 .

What is characteristic for this maximum drift configuration is an optimal combination of a strong overlapping of atom-molecule interaction spheres, and still significant asymmetry of the spheres' union. Increasing of the $a$ with $\sigma$ kept fixed would enhance the asymmetry of the spheres' union but decrease their overlapping, as for configuration $B$ in Fig. 5. Such a configuration is characterized by a smaller drift velocity, see Fig. 6. On the other hand, increasing the interaction spheres radius $\sigma$ with atomic cluster size $a$ kept constant would increase the spheres' overlapping but reduce the asymmetry of the spheres' union. Again, this results in a decrease of the cluster's drift velocity. These trends corroborate those we observed in the previous subsections for dimers and, according to (72), reflect dependence of the nonlinear dissipation coefficient $\gamma_{1}$ and the underlying correlation $C_{1}(t)$ on structural properties of the cluster.

\section{CONCLUSION}

In this paper we considered intrinsic ratchets as microscopic clusters of atoms interacting with bath molecules through given short-range potentials. The assumption that clusters atoms are connected by rigid bonds, adopted in 


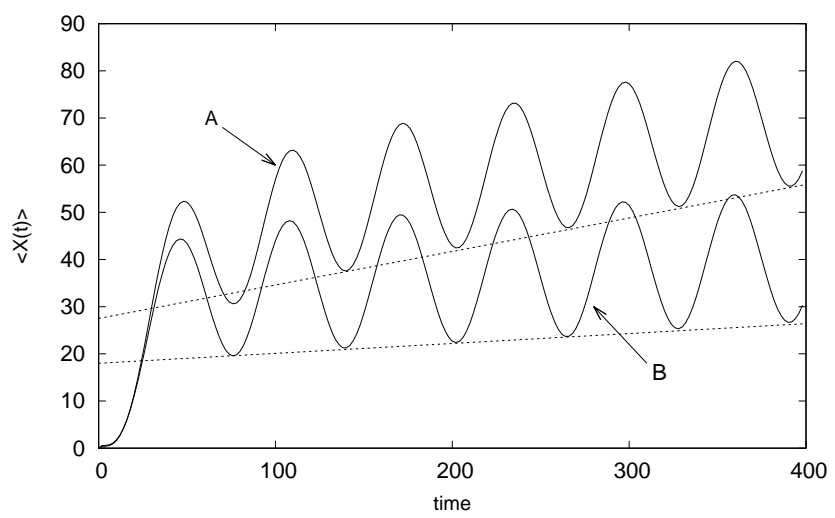

FIG. 6: The average displacement (solid lines) of two trimer configurations $A$ and $B$ shown in Fig. 5 activated by a harmonic external force. The slopes of tangent lines to minima (dashed) give the net velocity $V_{\text {net }}$ of a cluster. We found $V_{\text {net }} \approx 0.07$ for configuration $A$ and $V_{\text {net }} \approx 0.02$ for configuration $B$. The simulation parameters are: the molecule-atom mass ratio $m / M_{a}=0.075$ (molecule-trimer mass ratio $m / M=0.025$ ), external force frequency $\omega=0.1$ and amplitude $F_{0}=2$, squared harmonic bond frequency $\Omega^{2}=k / M_{a}=4.5$, bath density $\rho=0.2$.

the theoretical part of this paper, does not appear to be restrictive. The nonlinear Langevin equation for soft clusters can be derived in a similar way eliminating internal degrees of freedom of a cluster with a properly modified projector operator.

Compared to previous studies, our approach describes ratchets with broader types of asymmetry and also emphasizes the generality of the relevant nonlinear Langevin equation (57) and fluctuation-dissipation relations (52) and (58), particularly for the nonlinear dissipation coefficient $\gamma_{1}$. The latter is a key quantity which determines the drift velocity of a ratchet. While fluctuation-dissipation relations play important role in the theory, they are difficult to use and rarely exploited for a direct evaluation of dissipation coefficients. Instead, the linear dissipation coefficient $\gamma_{0}$ can be readily estimated experimentally or in simulation as the inverse momentum relaxation time, and the the nonlinear dissipation coefficient $\gamma_{1}$ can be determined comparing a measured or simulated net velocity $V_{\text {net }}$ with theoretical relations for $V_{\text {net }}$ obtained in this paper.

Our results suggest that a value of $\gamma_{1}$ and the underlying correlation function $C_{1}(t)$ depend in a delicate way on the composition of atom-molecule interaction spheres, rather than of the mere geometry of the cluster's atomic skeleton. For a symmetric cluster or a single atom $\gamma_{1}$ is zero, but it may be negligibly small for asymmetric clusters as well. On the one hand, $\gamma_{1}$ tends to increase for a larger overlapping of interaction spheres. On the other hand, the increase of the spheres' overlapping with their radius kept fixed diminishes the asymmetry of the spheres' union. The maximum value of $\gamma_{1}$ (and therefore a cluster's maximum drift velocity) is achieved for an optimal combination of the two factors, as for configurations $A$ on Figs. (2) and (5).

Let us also note a subtle role of the external force $F_{e x}(t)$ in the operation of intrinsic ratchets. On the one hand, this force "shakes" the system preventing it from reaching thermal equilibrium. For $F_{e x}(t)=F_{0} \sin \omega t$, the efficiency of this "shaking" is expected to increase with $\omega$. On the other hand, the particle's drift during one half-period of the force oscillation decreases with $\omega$. A nontrivial interplay of these two factors is that the drift velocity $V_{\text {net }}$ increases when $\omega$ decreases and, according to the presented theory, reaches a maximum in the adiabatic regime, i.e. when $F_{e x}(t)$ varies infinitely slow. Such behaviour is in contrast with many other types of ratchets activated by a modulated external potential, which cannot work arbitrary close to equilibrium [2, 3, 27]. Our simulations confirm qualitatively the theoretical dependence of $V_{n e t}$ on $\omega$ (and on the correlation time $\tau_{c}$, for a fluctuating external force), though the adiabatic regime is of course directly inaccessible in simulation.

The nonlinear Langevin equation obtained in this paper is of the same form as for the familiar problem of an adiabatic piston separating two gases of different temperatures [11]. The adiabatic piston problem can be formulated in a one-dimensional form and under certain model assumptions allows an analytical evaluation of relevant correlation functions and dissipation coefficients [1] . Then it might be tempting to develop a more simple model of intrinsic ratchets using a one-dimensional geometry of the piston problem. For instance, one may assume that the left and right sides of the piston are made of different materials, so that molecules of left and right gases (now at the same temperature) interact with the piston via different potentials. One might expect that a piston with such structural asymmetry would behave as an intrinsic ratchet, i.e. develop an average drift velocity when subjected to an external unbiased low-frequency force. Remarkably, we found no evidence for that. For a specific model where the thermal 
bath is a uniform ideal gas whose molecules interact with the piston via a parabolic potential (of different amplitudes for left and right piston's sides), analytical results of Ref. [11] predict that $\gamma_{1}=0$ and therefore there is no drift. Our simulations show no drift for other types of asymmetric potentials as well. This is perhaps not surprising considering the two sides of the piston as analogues of the dimer's atoms and recalling the importance of the overlapping of atom-molecule interaction regions as a condition for the drift. There is no such overlapping for the piston geometry, and therefore there is no drift.

Instead of the nonlinear Langevin equation, one can use an equivalent corresponding master equation for the velocity or momentum distribution function $f(P, t)$. To order $\lambda^{3}$ the master equation differ from the standard second order Fokker-Planck equation by involving the $P$-derivative of order three [10, 14, 16, 17]. A common worry is that according the Pawula theorem [28] a master equation of order higher than two may not preserve positivity of the distribution function. To answer this, it was emphasized elsewhere [29] that an approximate distribution function does not need to be positive for all values of its arguments. It may take a small negative values in far-tail regions and still be computationally useful. The terms with higher order derivatives are also of higher order in $\lambda$, and as long as one treats them consistently as perturbations, the results are meaningful and valid to a given order in $\lambda$. Several model calculations showed that an approximate distribution given by master equations of higher orders may be negative in the far-peripheral regions, which are computationally negligible since the distribution there is very small; on the other hand, for the region(s) where the distribution is large the corrections stemming from the higher derivative terms may be important and do not violate the distribution's positivity [28, 30 34]. In practice, instead of solving a master equation of higher order it is usually easier to get and solve perturbatively equations for the moments. In this form, the validity and usefulness of the method was demonstrated in many works, e.g. [1, 11, 12, 14, 18, 20, 21, 35].

\section{APPENDIX}

Let us show that for a symmetric Brownian particle consisting of a single atom interacting with molecules of the bath via a spherically symmetric potential, the correlation function $C_{1}(t)$ given by (52), and therefore the nonlinear damping coefficient $\gamma_{1}=\left(\beta / M^{2}\right) \int_{0}^{\infty} C_{1}(t) d t$, are zero. In that case the bath Hamiltonian $H_{0}$ (9) depends on position vectors of the particle $\mathbf{R}=(X, Y, Z)$ and molecules $\mathbf{r}_{i}=\left(x_{i}, y_{i}, z_{i}\right)$ only through the lengths of the difference vectors $\mathbf{q}_{i}=\mathbf{r}_{i}-\mathbf{R}$ and $\mathbf{q}_{i}-\mathbf{q}_{i^{\prime}}=\mathbf{r}_{i}-\mathbf{r}_{i^{\prime}}$. Under the inversion of the phase space

$$
\mathbf{p}_{i} \rightarrow-\mathbf{p}_{i}, \quad \mathbf{q}_{i} \rightarrow-\mathbf{q}_{i}
$$

the Hamiltonian $H_{0}$ and the Liouville operator $L_{0}=\left\{\cdots, H_{0}\right\}$ are invariant, while the operator $\frac{\partial}{\partial X}=\sum_{i} \frac{X-x_{i}}{\left|\mathbf{q}_{i}\right|} \frac{\partial}{\partial\left|\mathbf{q}_{i}\right|}$ is odd,

$$
H_{0} \rightarrow H_{0}, \quad L_{0} \rightarrow L_{0}, \quad \frac{\partial}{\partial X} \rightarrow-\frac{\partial}{\partial X} .
$$

According to (52), $C_{1}(t, \tau)=\int_{0}^{t}\langle A(t, \tau)\rangle d \tau$ where

$$
\langle A(t, \tau)\rangle=\frac{1}{Z_{0}} \int e^{-\beta H_{0}} A(t, \tau) \prod_{i} d \mathbf{q}_{\mathbf{i}} d \mathbf{p}_{\mathbf{i}}
$$

is the average of the dynamical function

$$
A(t, \tau)=F e^{L_{0}(t-\tau)} \frac{\partial}{\partial X} F_{0}(\tau)=\frac{\partial H_{0}}{\partial X} e^{L_{0}(t-\tau)} \frac{\partial}{\partial X} e^{L_{0} \tau} \frac{\partial H_{0}}{\partial X} .
$$

Taking into account (A2) one notices that under the inversion transformation (A1) the function $A$ is odd, $A \rightarrow-A$. Making in the integral (A3) the substitution $\mathbf{q}_{i}=-\mathbf{q}_{i}^{\prime}$ and $\mathbf{p}_{i}=-\mathbf{p}_{i}^{\prime}$, and taking into account the symmetry properties of $H_{0}$ and $A$, one finds $\langle A(t, \tau)\rangle=-\langle A(t, \tau)\rangle=0$, and $C_{1}(t)=\int_{0}^{t}\langle A(t, \tau)\rangle d \tau=0$.

[1] M. van den Broek, R. Eichhorn, and C. Van den Broeck, EPL 86, 30002 (2009).

[2] P. Reimann, Phys. Rep. 361, 57 (2002).

[3] P. Hanggi and F. Marchesoni, Rev. Mod. Phys. 81, 387 (2009).

[4] G. Costantini, U. Marini Bettolo Marconi, and A. Puglisi, Phys. Rev. E 75, 061124 (2007); Europhys. Lett. 82, 50008 (2008). 
[5] B. Cleuren and C. van den Broeck, EPL 77, 50003, (2007).

[6] J. A. Fay, Phys. Rev. E 56, 3460 (1997).

[7] F. Ahmadpoor, L. Liu, P. Sharma, J. Mech. Phys. Solids 78, 110 (2015).

[8] S. Kim and I. Oppenheim, Physica 57, 469 (1972).

[9] H. Mori, H. Fujisaka, and H. Shigematsu, Prog. Theor. Phys. 51, 109 (1974).

[10] N. G. Van Kampen and I. Oppenheim, Physica A, 138, 231 (1986).

[11] A. V. Plyukhin and J. Schofield, Phys. Rev. E 69, 021112 (2004).

[12] A. V. Plyukhin and A. M. Froese, Phys. Rev. E 76, 031121 (2007).

[13] M. F. Gelin and D. S. Kosov, J. Chem. Phys. 126, 244501 (2007).

[14] P. Meurs, C. Van den Broeck, and A. Garcia, Phys. Rev. E 70, 051109 (2004).

[15] N. G. van Kampen, Stochastic Processes in Physics and Chemistry, 3rd ed., North-Holland, Amsterdam, 2007.

[16] C.T.J. Alkemade, N.G. van Kampen and D.K.C. Mac Donald, Proc. Roy. Soc. A 271, 449 (1963).

[17] A. V. Plyukhin, Physica A 351, 198 (2005).

[18] A. V. Plyukhin, Phys. Rev. E 84, 061124 (2011).

[19] C. Van den Broeck, R. Kawai, and P. Meurs, Phys. Rev. Lett. 93, 090601 (2004).

[20] C. Van den Broeck, P. Meurs, and R. Kawai, New J. Phys. 7, 10 (2005).

21] C. Van den Broeck and R. Kawai, Phys. Rev. Lett. 96, 210601(2006).

[22] M. van den Broek and C. Van den Broeck, Phys. Rev. E 78, 011102 (2008).

[23] A. Fruleux, R. Kawai, and K. Sekimoto, Phys. Rev. Lett. 108, 160601 (2012).

[24] M. Itami and S. Sasa, J. Stat. Phys. 158, 37 (2015).

[25] M. E. Tuckerman, Statistical Mechanics: Theory and Molecular Simulation, Oxford University Press, New York, 2010.

[26] R. M. Mazo, Brownian Motion, Oxford University Press, New York, 2009.

[27] J. M. R. Parrondo and B. J. de Cisneros, Appl. Phys. A 75, 179191 (2002).

[28] H. Risken, The Fokker-Planck Equation, 2nd ed., Springer, Berlin, 1989.

[29] See, e.g., section 4.6 in Ref. [28] and section X.6 in Ref. [15].

[30] H. Risken and H. D. Vollmer, Z. Phys. B 35, 313 (1979).

[31] Y. Hasegawa and M. Arita, Physica A 390, 1051 (2011).

[32] R. Grima, P. Thomas, and A. V. Straube, J. Chem. Phys. 135, 084103 (2011).

[33] R. Grima and P. Thomas, Phys. Rev. E 92, 012120 (2015).

[34] C. Cianci, F. Di Patti, and D. Fanelli, Europhys. Lett. 96, 50011 (2011).

[35] C. Cianci, D. Schnoerr, A. Piehler, and R. Grima, J. Phys. A: Math. and Theor. 50, 395003 (2017). 